Minimizing total flow time in the single-machine scheduling problem with periodic maintenance

Journal of the Operational Research Society (2012) 63, 567. doi:10.1057/jors.2011.71

As we observe, the numerical example proposed by Chen (2006) is incorrect because the author does not follow the steps of their proposal heuristic algorithm to illustrate example 1. According to Step 1(i) of the heuristic algorithm proposed by Chen, we should have three batches each with a total processing time of $T=8$. For instance, the first batch may contain the first three jobs, the second batch may contain the next three jobs, and the third batch may contain the next three jobs. According to Step 1(ii), we then have another batch with a total processing time of $T-4=4$. If the heuristic algorithm performs exactly as we have just done, then the initial schedule, $\sigma_{\pi}$, after Step 1 can be presented as shown in Table 1 .

After Step 3, we obtain $F\left(\sigma_{\pi}\right)=164$, see Table 2 .

It can be checked that the schedule presented in Table 2 is the final schedule. In other words, Steps $4-7$ of the heuristic algorithm will not change the schedule.

Moreover, Knödel (1981) does not indicate the method of grouping jobs as stated in Step 1(i) of the heuristic, see Knödel (1981) for details.

\section{Response to $\mathrm{Xu}$ and Xiong}

Journal of the Operational Research Society (2012) 63, 567. doi: $10.1057 /$ jors. 2011.70

Using Steps 1-3 of the heuristic, we can obtain many schedules. One of the schedules is the one presented in Table 6 (in the original article).

\begin{tabular}{lrrrrrrrrrrrrr}
\hline$J_{i}$ & $J_{3}$ & $J_{4}$ & $J_{9}$ & $J_{5}$ & $M_{1}$ & $J_{8}$ & $J_{6}$ & $J_{10}$ & $M_{2}$ & $J_{1}$ & $J_{2}$ & $M_{3}$ & $J_{7}$ \\
\hline$p_{i}$ & 1 & 2 & 2 & 3 & 1 & 3 & 4 & & 3 & 4 & & 5 \\
$C_{i}$ & 1 & 3 & 5 & 8 & 11 & 14 & 18 & & 23 & 27 & & 35 \\
$F(S)$ & 1 & 4 & 9 & 17 & 28 & 42 & 60 & & 83 & 110 & & 145
\end{tabular}

In order to facilitate a better understanding of the heuristic, we chose an initial table (Table 2, in the original article) to match the steps of the heuristic. This
Table 1 Initial schedule after Step 1 of the heuristic for example 1 (in h)

\begin{tabular}{lrrrrrrrrrrrrr}
\hline$J_{i}$ & $J_{1}$ & $J_{2}$ & $J_{3}$ & $M_{1}$ & $J_{4}$ & $J_{5}$ & $J_{6}$ & $M_{2}$ & $J_{7}$ & $J_{8}$ & $J_{9}$ & $M_{3}$ & $J_{10}$ \\
\hline$p_{i}$ & 3 & 4 & 1 & & 2 & 3 & 3 & & 5 & 1 & 2 & & 4 \\
$C_{i}$ & 3 & 7 & 8 & & 12 & 15 & 18 & & 25 & 26 & 28 & & 34 \\
$F(S)$ & 3 & 10 & 18 & & 30 & 45 & 63 & & 88 & 114 & 142 & & 176 \\
\hline
\end{tabular}

Table 2 Initial schedule after Step 3 of the heuristic for example 1 (in h)

\begin{tabular}{lrrrrrrrrrrrrr}
\hline$J_{i}$ & $J_{3}$ & $J_{1}$ & $J_{2}$ & $M_{1}$ & $J_{4}$ & $J_{5}$ & $J_{6}$ & $M_{2}$ & $J_{8}$ & $J_{9}$ & $J_{7}$ & $M_{3}$ & $J_{10}$ \\
\hline$p_{i}$ & 1 & 3 & 4 & & 2 & 3 & 3 & & 1 & 2 & 5 & & 4 \\
$C_{i}$ & 1 & 4 & 8 & & 12 & 15 & 18 & & 21 & 23 & 28 & & 34 \\
$F(S)$ & 1 & 5 & 13 & & 25 & 40 & 58 & & 79 & 102 & 130 & & 164 \\
\hline
\end{tabular}

\section{References}

Chen WJ (2006). Minimizing total flow time in the single-machine scheduling problem with periodic maintenance. $J$ Opl Res Soc 57: $410-415$.

Knödel W (1981). A bin packing algorithm with complexity O(n log n) and performance 1 in the stochastic limit. Math Found Comput Sci 118: 369-378.

East China Institute of Technology

$\mathrm{D} \mathrm{Xu}$ S Xiong

is because the schedule with some potential positions (Theorems 2 and 3 can be applied) is usually produced in our testing.

Therefore, Table 1 (in the Viewpoint contributed by Xu and Xiong, ...) constitutes one of the above-discussed schedules. We think that Table 2 (by Xu and Xiong ...) is not the final schedule and can be improved by using the heuristic.

Finally, we need to remark that we (not Knodel, 1981) use the bin-packing technique to group jobs; this technique was just mentioned but not used by Knodel.

\section{Reference}

$\mathrm{Xu} \mathrm{D}$ and Xiong $\mathrm{S}(\ldots)$. Minimizing total flow time in the singlemachine scheduling problem with periodic maintenance. $J \mathrm{Opl}$ Res Soc.

China University of Technology (Taiwan)

WJ Chen 